\title{
Implementation of XpertMalTyph: An Expert System for Medical Diagnosis of the Complications of Malaria and Typhoid
}

\author{
S.A. Fatumo ${ }^{1}$, Emmanuel Adetiba ${ }^{2}$, J.O.Onaolapo ${ }^{3}$ \\ ${ }^{1}$ (Computer and Information Science Department, College of Science and Technology, Covenant University, \\ Canaan land, Ota, Nigeria) \\ ${ }^{2,3}$ (Electrical and Information Engineering Department, College of Science and Technology, Covenant \\ University, Canaan land, Ota, Nigeria)
}

\begin{abstract}
The dearth of medical experts in the developing world has subjected a large percentage of its populace to preventable ailments and deaths. Also, because of the predominant rural communities, the few medical experts that are available always opt for practice in the few urban cities. This consequently puts the rural communities at a disadvantage with respect to access to quality health care services. In this work, we designed and implemented XpertMalTyph; a novel medical diagnostic expert system for the various kinds of malaria and typhoid complications. A medical diagnostic expert system uses computer(s) to simulate medical doctor skills in diagnosis of ailments and prescription of treatments, hence can be used to provide the same service in the absence of the experts. XpertMalTyph is based on JESS (Java Expert System Shell) programming because of its robust inference engine and rules for implementing expert systems.
\end{abstract}

Keywords: XpertMalTyph, medical informatics, diagnosis, malaria, typhoid.

\section{Introduction}

The fundamental reason for visiting a hospital is to get adequate treatment and attention without delay from the best hands. No patient wants to be a specimen for a doctor especially one that has not improved on the knowledge gained since graduation from the medical school and at the same time, patients do not like to be delayed in receiving treatment for whatever reason. But all these aforementioned have become the order of the day in most hospitals; especially in developing countries. Patients cannot get to see the doctor at the appropriate time and there are some patients that find it difficult communicating their health issues and the doctor in turn has no time to keep asking the probable questions [1].

There are also cases of patients that don't like going to the hospital for various reasons such as the smell of drugs, some unwelcoming nurses, the long wait for the doctor or simply laziness. It becomes disheartening as the death rate in most developing countries increases even due to minor ailments that could have being treated [2].

Another issue that has posed a problem in medicine in the developing world is the inadequate infusion of technology to aid the activities involved in the practice. Since technology is a very useful tool that aids every kind of process resulting in increase of efficiency, effectiveness and in turn productivity, automating some of the processes involved in attending to patients so as to reach a wide number of them at the same time from different locations would be of immense benefit [3].

\subsection{Artificial Intelligence and Expert Systems}

Intelligence can be defined as the ability to learn and understand, to solve problems and to make decisions. However, Artificial Intelligence (AI) is the display of intelligence by machines, and its main goal as a field is to make machines do things that would require intelligence if done by humans. The central problems of AI include such traits as reasoning, knowledge, planning, learning, communication, perception and the ability to move and manipulate objects. In the early $90 \mathrm{~s}$, AI achieved its greatest feats in practical application despite all its previous setbacks. It was successfully applied in logistics, data mining and medical diagnosis. However, AI success started being revived with the commercial success of expert systems [4].

Expert System is a form of Artificial Intelligence program that simulates the knowledge and analytical skills of one or more human experts. Conventional expert systems are built for a very narrow domain with clearly defined expertise. It makes the system performance fully dependent on the right choice of experts. Although a common strategy is to find just one expert, when a more complex expert system is being built or when expertise is not well defined, multiple experts might be needed [5]. Fig. 1 shows the generic block diagram of medical expert systems. XpertMalTyph is a medical expert system for diagnosis of the complications of malaria and typhoid. 


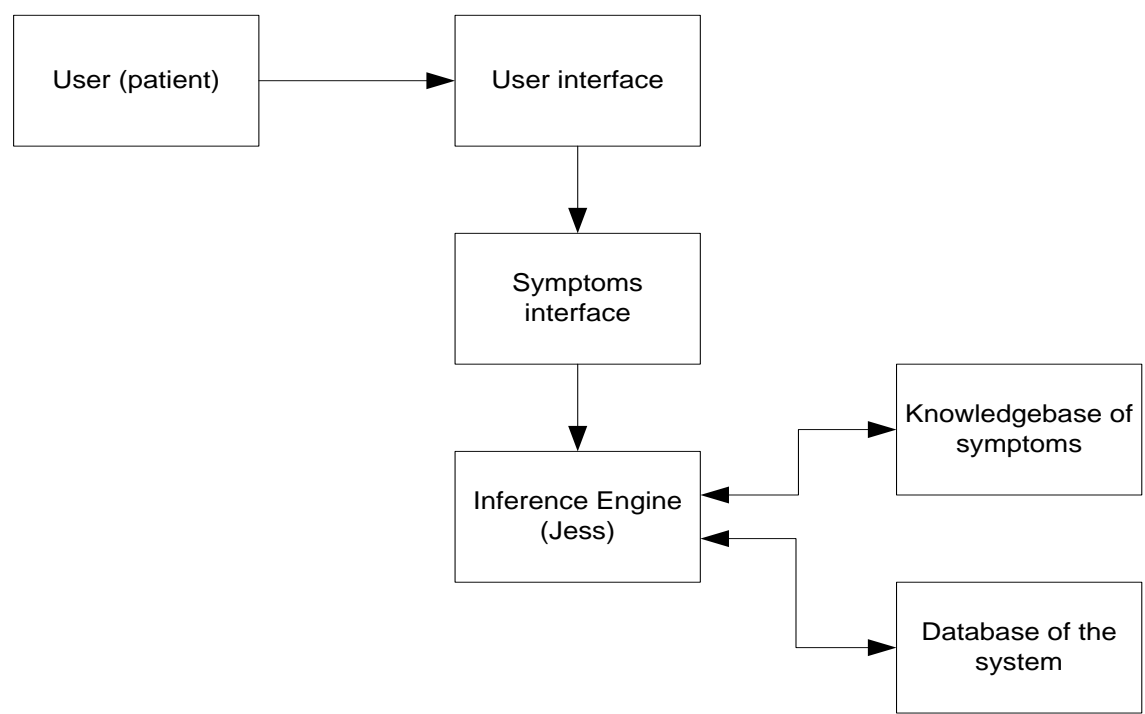

Fig.1: Medical Expert System Architecture

\subsection{Malaria and Typhoid Diagnosis}

Malaria causes significant morbidity and mortality worldwide. In developing nations, scarce resources lead to inadequate diagnostic procedures. Malaria can result in anemia (a decreased number of red blood cells). The remains of the destroyed red blood cells clump together and cause blockages in the blood vessels. This can result in brain damage or kidney damage, which is potentially fatal. A particularly serious, potentially lifethreatening, form of malaria parasite is called Plasmodium falciparum [6].

In developing countries, limited resources may cause clinical and laboratory misdiagnosis of malaria. Diagnosis of malaria involves identification of malaria parasite or its antigens/products in the blood of the patient. The most commonly used diagnostic test for malaria is microscopy to detect parasites in stained blood films. Thick blood films are used in routine diagnosis and as few as one parasite per $200 \mu \mathrm{l}$ blood can be detected. The method can be used to differentiate between different parasite species and stages of the life cycle. Although it has good sensitivity and allows species identification and parasite counts, it is time consuming, requires microscopy expertise and maintenance of equipment.

Microscopy with fluorescent stains (QBC), dipstick antigen detection of HRP2 and pLDH (Parasight-F, ICT Malaria Pf, OptiMAL), polymerase chain reaction assays and some automated blood cell analyzers offer new approaches with emphasis on clinical relevance and their potential to complement conventional microscopy, especially in countries with imported malaria. People with uncomplicated malaria often have fever, or a recent history of fever, headache, vomiting, watery diarrhea, anemia and an enlarged spleen. In children, additional symptoms include convulsions and cough.

Similarly, a bacteria called Salmonella typhi (S. typhi) is responsible for typhoid. S. typhi may be spread by consuming contaminated water, beverages and food, after which the bacteria enter the intestines and then the bloodstream, where they may spread to other body parts. Initial typhoid symptoms include malaise, headache, diarrhea (or constipation), sore throat, fever as high as $104^{0} \mathrm{~F}$, as well as a rash. Diagnosis is made by any blood, bone marrow or stool cultures and with the Widal test. In epidemics and less wealthy countries, after excluding malaria, dysentery or pneumonia, a therapeutic trial time with chloramphenicol is generally undertaken while awaiting the results of Widal test and cultures of the blood and stool [7].

However, Rapid and accurate diagnosis of malaria and typhoid is integral to the appropriate treatment of affected individuals and in preventing the further spread of infection in the community. XpertMalTyph developed in this study provides opportunities to patients in developing countries to identify when they have malaria and/or typhoid and seek solutions in the comfort of their homes.

\subsection{Literature Review of Existing Medical Expert Systems}

GIDEON is an expert system that is a very useful resource for medical practitioners because it provides decision support for generating a differential diagnosis. It is used for difficult-to-identify infectious disease for patients with travel histories. It clarifies unusual disease patterns and helps to plan effective treatment [8]. To assist medical professionals with diagnosis, GIDEON generates a ranked list of possible diseases based on signs, symptoms, laboratory tests and exposure history for any infectious disease, in any country of the world. As an option, users can also simulate all possible Bioterrorism scenarios. An additional Microbiology module is designed to identify or study any of the more than 2,500 known human bacteria, viruses, fungi and parasites [9]. 
MYCIN, a medical diagnostic expert system was designed at Stanford University in 1972. MYCIN is intended to provide physicians with advice about the diagnosis and drug therapy for bacterial infections. It identifies the organisms responsible for an infection from information concerning the patient's symptoms and test results. It was developed with LISP as the doctoral dissertation of Edward Shortliffe under the direction of Bruce Buchanan, Stanley N. Cohen and others. It arose in the laboratory that had created the earlier Dendral expert system, but emphasized the use of judgmental rules that had elements of uncertainty (known as certainty factors) associated with them [10]. This expert system was designed to identify bacteria causing severe infections, such as bacteremia and meningitis, and to recommend antibiotics, with the dosage adjusted for patient's body weight. The name was derived from the antibiotics themselves, as many antibiotics have the suffix "mycin".

Several other medical expert systems exist as well. A notable example is STENO, which is based on causal probabilistic networks as detailed in[11].

\subsection{System Design}

\section{METHODS}

The system design of XpertMalTyph (our medical diagnostic expert system) serves as the blueprint for a robust implementation. The design is divided into stages:

i.) Logical design: This is an implementation-independent design which lays out the components of the system and their relationships to one another as they will appear to the users. It describes input/output procedures, processing functions to be performed, business procedures, data models and controls. The system is comprised of logical modules such as registration, login, diagnosis and contacts.

ii.) Physical design: This is an implementation-dependent design which is concerned with those aspects of the system that are dependent on the implementation platform. At this stage, the abstract logical model is translated into the specific technical design for the system. It produces the specification for the hardware, software, physical storage, input/output media and etc. The physical design gives structure in terms of look \& feel and functionality to the logical design of the system. A system module block diagram is shown in Figure 2.

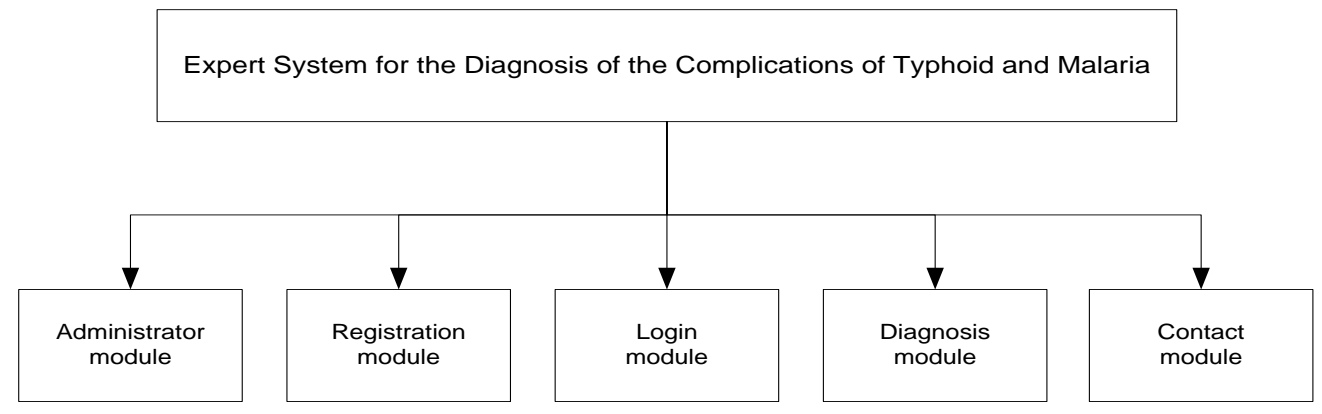

Fig.2: Block diagram of system modules

\subsection{System Modeling}

A formal model of the proposed system is built using Unified Modeling Language (UML). The UML is a modeling system which provides a set of conventions that are used to describe a software system in terms of objects [12]. It offers diagrams that provide different perspective views of the system parts. The UML diagrams used to model the system are; Use Case, Sequence and Activity diagrams.

A use case diagram graphically depicts the interactions between the system, the external system and the user. Use case diagrams play a major role in system design because it acts as a roadmap in constructing the structure of the system; it also defines who will use the system and in what way the user expects to interact with the system. The Use Case diagram for our expert system is shown in Figure 3. 


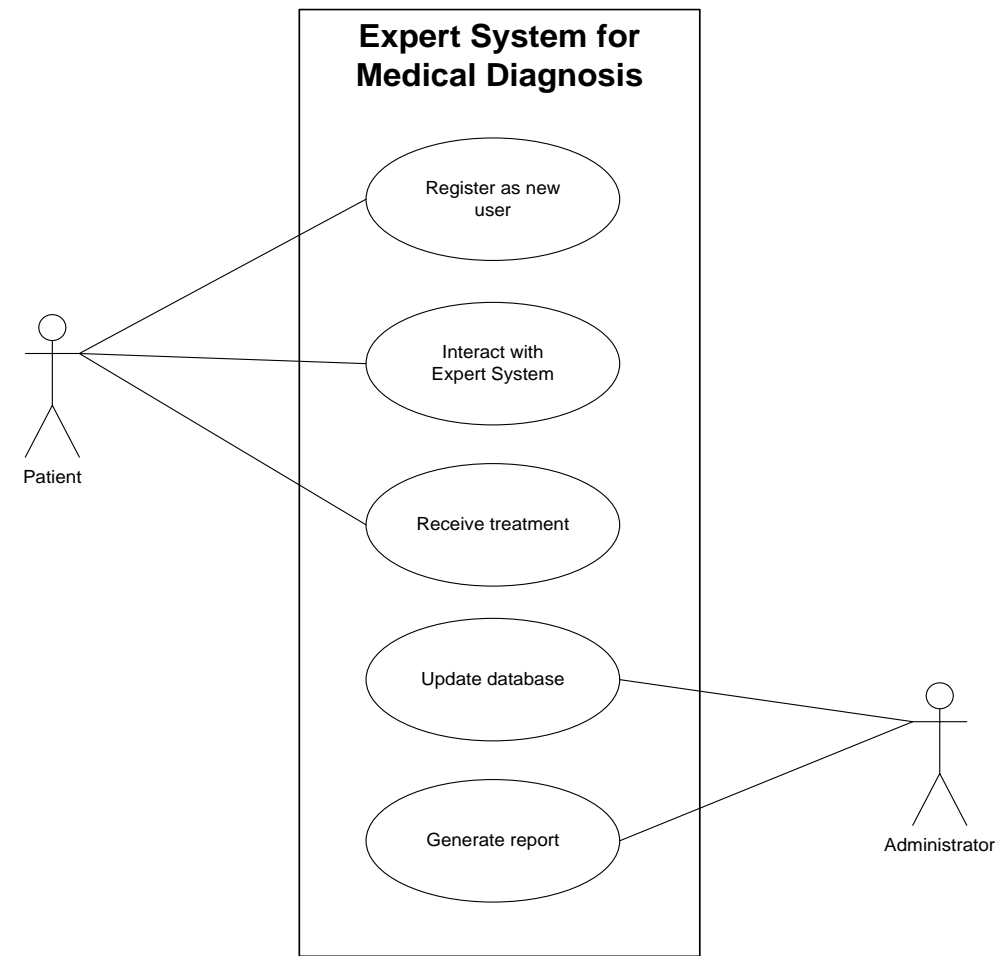

Fig.3: Use Case diagram of XpertMalTyph

Table 1 represents the glossary for the use case in Fig. 3.

Table 1: Use Case description

\begin{tabular}{|l|l|l|}
\hline \multicolumn{1}{|c|}{$\begin{array}{c}\text { USE CASE } \\
\text { NAME }\end{array}$} & \multicolumn{1}{|c|}{ DESCRIPTION } & \multicolumn{1}{c|}{$\begin{array}{c}\text { ACTOR(S) } \\
\text { INVOLVED }\end{array}$} \\
\hline $\begin{array}{l}\text { Register into } \\
\text { Expert System }\end{array}$ & $\begin{array}{l}\text { This describes the first event that must occur for a user to have full } \\
\text { access to the Medical Expert System }\end{array}$ & Patient \\
\hline $\begin{array}{l}\text { Interacts with the } \\
\text { Expert System }\end{array}$ & $\begin{array}{l}\text { This describes the event where the user interacts with the system, } \\
\text { that is, gives response to the questions asked by the Expert System. }\end{array}$ & Patient \\
\hline Receives treatment & $\begin{array}{l}\text { This use case describes the scenario where the user is being } \\
\text { diagnosed and given treatment. }\end{array}$ & Patient \\
\hline Update database & $\begin{array}{l}\text { This describes the scenario where the Administrator updates the } \\
\text { database in terms of the symptoms and prescription tables. }\end{array}$ & Administrator \\
\hline Generate Report & Reports of daily use of System are generated & Administrator \\
\hline
\end{tabular}

Activity diagrams graphically represent the performance of actions or sub-activities and the transactions that are triggered by the completion of the actions or sub-actions. It is a means of describing the workflow of activities. The purpose of an activity diagram is to provide a view of flows and what is going on inside a Use Case [13]. The Activity diagram for XpertMalTyph Use Case is shown in Fig. 4 while Fig. 5 shows the Sequence diagram for the register Use Case. 


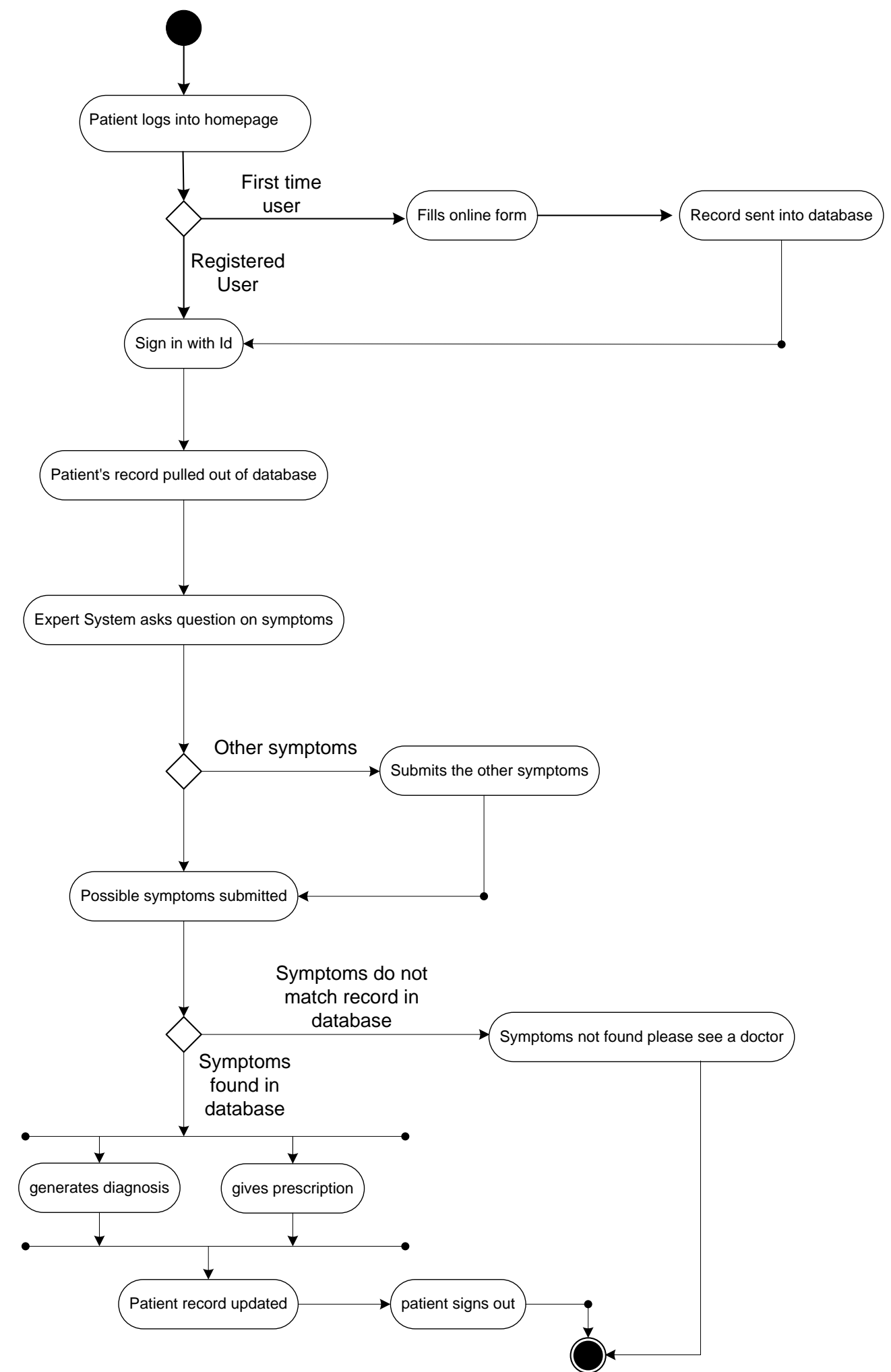

Fig. 4: Activity diagram of XperMalTyph 


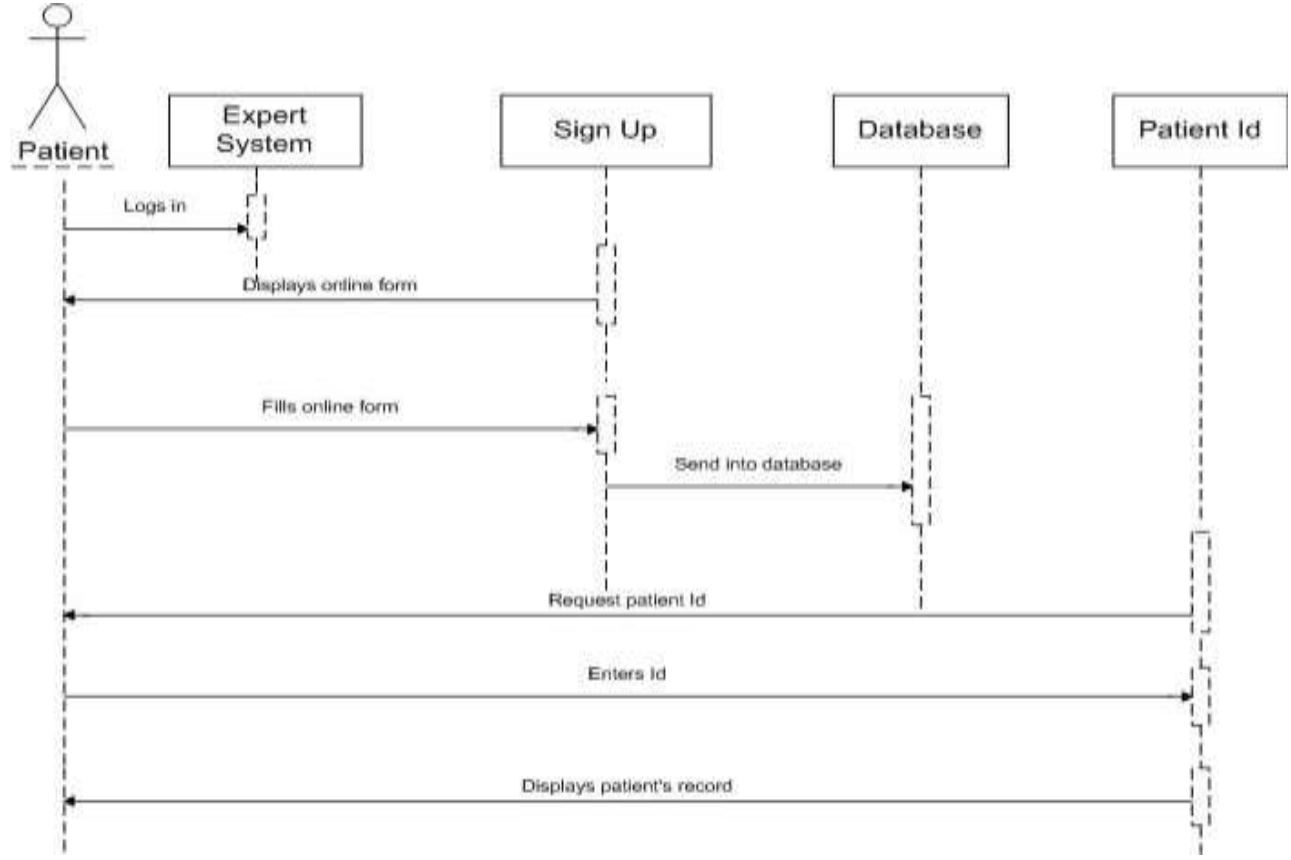

Fig. 5a: Sequence diagram for 'Register with Expert System' Use Case

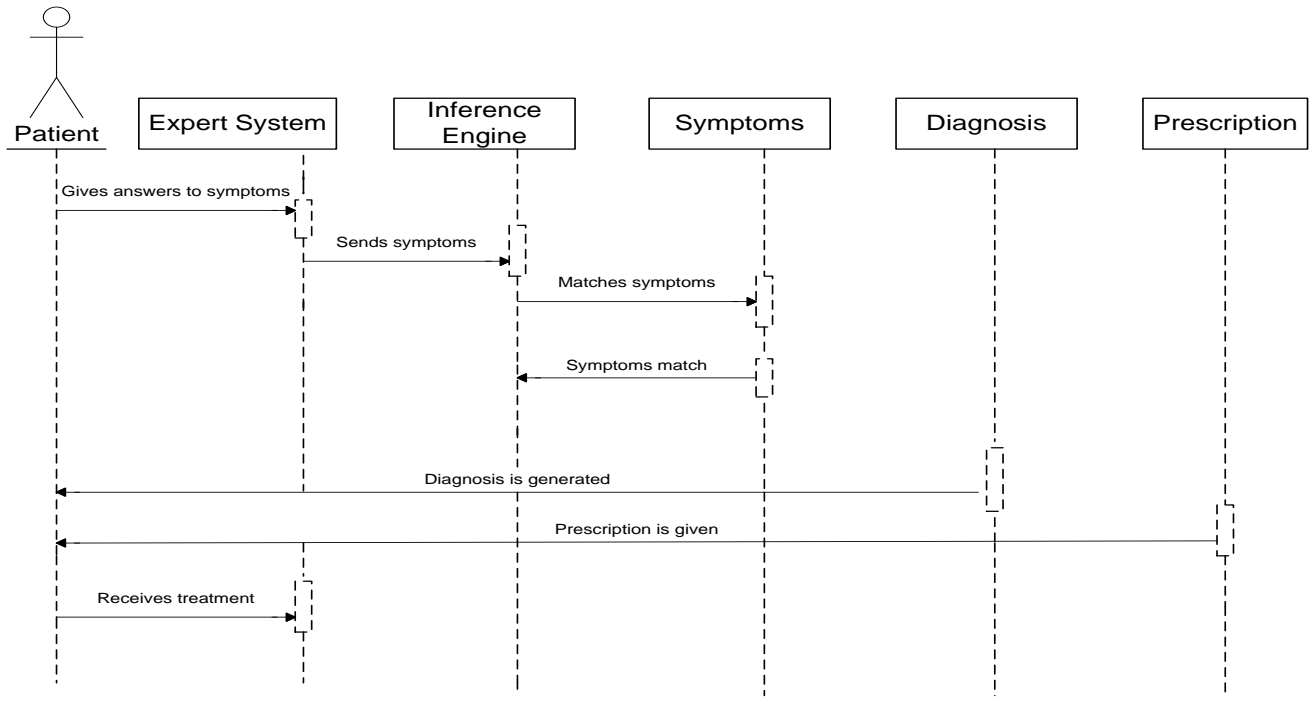

Fig.5b: Sequence diagram for 'Register with Expert System' Use Case

\subsection{Tools and Data Collection}

The process of developing our medical diagnostic system requires several sessions with a professional doctor so as to understand and get the details of malaria and typhoid diseases. The formal model of the system was developed using Unified Modeling Language (UML) so as to have a detailed description of the functionality of the system and understand the basic requirements.

Java Expert System Shell (JESS) was used to implement the expert module of our medical diagnostics system. JESS is an Expert System shell created with Java and for Java which is used for developing Expert Systems of all kinds. The rule engine of any Expert System is best developed using JESS because it allows for ease of processing of rules [14].

Java programming language was used as the implementation tool because of its Java Server Page (JSP) which makes the Expert System a web-based application. The database engine used was MySQL since it is robust and can accommodate a large amount of data. In addition, MySQL easily integrates with Java and JESS.

\section{Results}

The XperMalTyph Expert System has been designed to be user friendly and it is easy to navigate. The various modules used have been integrated from a single web interface. The user can easily access the whole 
application from the home page where there is menu bar from which the user can perform the desired functions as illustrated in the Use Case diagram (Fig. 3).

\section{Conclusions}

The medical diagnostic expert system for the various complications of malaria and typhoid has been designed to help patients and assist doctors not to replace them. There are times that some symptoms that an individual has might be taken for granted, that is, they might just be overlooked and the individual will not see a need to visit the hospital. This means that instead of a patient consulting the doctor for mild cases of malaria and typhoid complications, the patient can receive treatment even from the house through the Medical Diagnostic Expert system. Work is in progress to enhance this expert system by implementing the system with the hybrid of expert system and artificial neural networks (ANN).

\section{References}

[1] Cleary P.D., Edgman-Levitan S., Roberts M., et al. Patients evaluate their hospital care: a national survey. Health Affairs. 10 (4), 1991, 254-67.

[2] Coulter A., Cleary P.D., Patients' experiences with hospital care in five countries, Health Aff (Millwood ), 20, 2000, 244-52.

[3] Frost L.J., Reich M.R.,. Creating access to health technologies in poor countries, Health Affairs, 28, 2009,962-73.

[4] Russell S.J., Norvig P., Artificial Intelligence: A Modern Approach. 2nd ed( Upper Saddle River, New Jersey: Prentice -Hall, 2003).

[5] Negnevitsky M. Artificial Intelligence, A guide to Intelligent Systems(Harlow, England: Addison Wesley, 2005).

[6] Malaria Site [Internet]. Clinical features of Malaria; c2011-13 [cited 2012 Sep 20]. Available from: http://www.malariasite.com/

[7] Ryan K.J., Ray C.G. Sherris Medical Microbiology. 4th ed.( New York: McGraw Hill, 2004).

[8] GIDEON: The world's premier global infectious diseases database; c1994-2012 Available from: http://www.gideononline.com/

[9] Sliwa J., Scientist study bacterial communities inside us to better understand health and disease, 2008 June 3, Available from: http://www.eurekalert.org/pub_releases/2008-06/asfm-ssb052908.php

[10] Fisher J . MYCIN Clinical Decision Support System . Available from: http://neamh.cns.uni.edu/MedInfo/mycin.html

[11] Andersen L.R., Krebs J.H., Andersen .J.D., STENO: An Expert System for Medical Diagnosis based on Graphical Models and Model Search, Journal of Applied Statistics, 18 (1). 1991, 139-53.

[12] Miller R. Practical UML: A Hands-On Introduction for Developers, Available from: http://edn.embarcadero.com/article/31863

[13] Ericsson M. Activity Diagrams: What They Are and How to Use Them, Available from: http://www.ibm.com/developerworks/rational/library/2802.html

[14] Friedman-Hill E.J.,Jess, The Java Expert System Shell, Available from: http://synotix.home.xs4all.nl/robbieng/docs/jess43doc.html 